EPiC Series in Engineering
Volume 1, 2018, Pages 26-30
ReVo 2017: Laval Virtual ReVolution 2017
"Transhumanism++"

\title{
The Holographic Human for surgical navigation using Microsoft HoloLens
}

\author{
Tomoki Itamiya ${ }^{1^{* \dagger}}$ and Toshinori Iwai ${ }^{2}$ and Tsuyoshi Kaneko ${ }^{3}$ \\ ${ }^{1}$ Aichi University of Technology, Gamagori, Japan \\ ${ }^{2}$ Yokohama City University Hospital, Yokohama, Japan \\ ${ }^{3}$ National Center for Child Health and Development, Setagaya-ku, Japan \\ itamiya@g.aut.ac.jp, iwai104oams@yahoo.co.jp, kaneko-t@ncchd.go.jp
}

\begin{abstract}
In surgical navigation, to accurately know the position of a surgical instrument in a patient's body is very important. Using transparent smart glasses is very useful for surgical navigation because a surgeon does not need to move his/her line of sight from the operative field. We propose a new application software development method that is able to show a stereoscopic vision of highly precise 3D-CG medical models and surgical instruments using transparent smart glasses for surgical navigation. We used Mixed Reality (MR) which is a concept exceeding Augmented Reality (AR) by using Microsoft HoloLens. In Mixed Reality, persons, places, and objects from our physical and virtual worlds merge together in a blended environment. Unlike competitive models, HoloLens can recognize surrounding with a front-facing cameras and 3D depth sensors. External markers and sensors are not required for surrounding recognition. Once a 3D-CG medical model is placed in a blended environment, it is fixed to the place and does not move on its own. Therefore, we can see a stereoscopic vision of a precise medical model projected into our surrounding such as a holographic human. A holographic human is as if he/she is there, which is a special immersive experience we have never felt before. A holographic human can not only be seen, but also can be moved by user's hand gestures and interactive manipulation is possible. A holographic human and 3D-CG surgical instrument can be displayed simultaneously in a blended environment. The movement of 3D-CG surgical instruments can be linked with actual surgical instruments in the operation room. In the operation room, the holographic human is superimposed on the actual patient position. Since the positional relationship between the holographic human and surgical instruments is clear because it is overlapping, so it is very useful for surgical navigation. Multiple persons can see one holographic human at the same time using multiple HoloLenses. We developed the holographic human application software for surgical navigation using Unity and Vuforia, which are a development software and a library. A holographic vision
\end{abstract}

\footnotetext{
${ }^{*}$ Masterminded EasyChair and created the first stable version of this document

${ }^{\dagger}$ Created the first draft of this document
} 
of a 3D-CG medical model made from an actual patient's CT/MRI image data is possible using our application software development method. A user can make the application software within only five minutes by preparing 3D-CG medical model file for instance STL. Therefore, surgeon dentists and clinical staff can make the holographic human content easily by themselves. As a result, the method can be utilized daily for routine medical treatment and education.

\section{Introduction}

The rapid development of new software and hardware tools for computer assisted planning and guidance of certain surgical procedures gives new perspectives in modern surgery. Especially craniomaxillofacial surgeons are confronted with complex anatomy and close spatial relationship of vital structures (for instance a brain). Cranio-maxillofacial surgery requires highly precise operations on a surgical target (for instance a tumor) that is often hidden by surrounding structures that must remain undamaged during the procedure. And, for facial cosmetic reasons the incision line has to be small, so surgeons have difficulty grasping the precise positional relationship between the patient's surgical target and surgical instrument from the outside. In surgical navigation, to accurately grasp the position of a surgical instrument in a patient's body is very important. In a conventional surgical navigation system[1], a surgeon had to look at the monitor in a different position from the surgical field, which made a surgeon tired. Virtual planning software and navigation systems using AR technology are widely used by cranio-maxillofacial surgeons[2][3][4][5]. Using AR is very useful because a surgeon does not need to move his/her line of sight from the operative field. However, substantial room for improvement remains. The video-based see-through system[2][3][5] has limitations on improving the resolution of video and the accuracy of alignment between a 3D-CG model and the real patient. The use of a halfsilvered mirror[4] has a negative effect on the use of surgical instruments in the operative field. Using a transparent smart glasses[6] may solve these problems, but the graphic performance and the precise alignment performance between a 3D-CG medical model and a patient were not satisfactory.

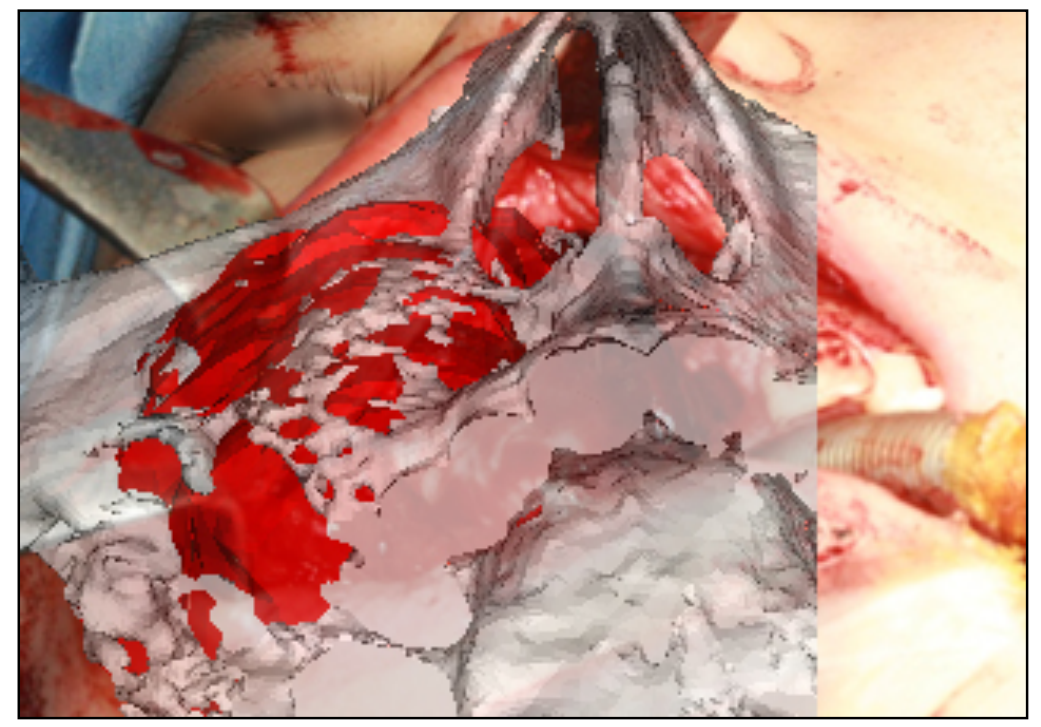

Figure 1: Example of surgeon's field of view when using AR transparent smart glasses in surgery 


\section{Our Method}

We proposed a new application software development method that is able to show a stereoscopic vision of highly precise 3D-CG medical models and surgical instruments using Microsoft HoloLens[7]. Our method has three steps to make the application software for Microsoft HoloLens. The first step is making a 3D-CG medical model of the data (STL) from CT/MRI 2D image data (DICOM) using Mimics[8]. The second step is to convert the STL file to fbx or obj data format file which enable the file to use with Unity[9]. The third step is making Mixed Reality application software with Unity and in some cases using Vuforia[10]. A user can make the application software within only five minutes by preparing STL. Therefore, surgeons dentists and clinical staff can make the holographic human content easily by themselves. As a result, the method can be utilized daily for routine medical treatment and education.

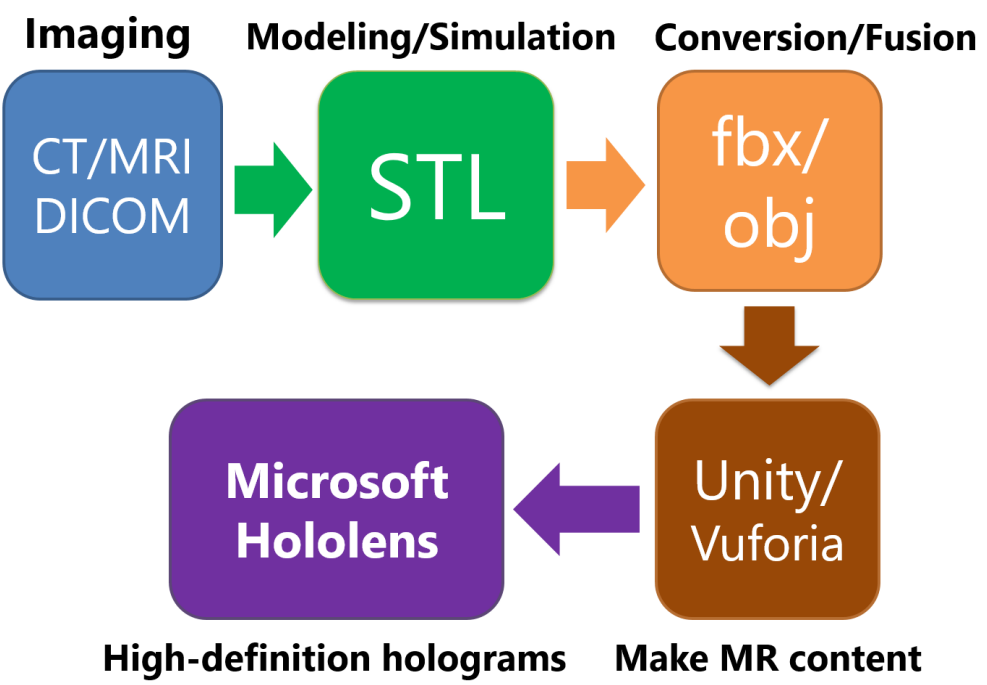

Figure 2: Structure of our method

\subsection{Overview of our software}

Our software for Microsoft HoloLens enables us to see a stereoscopic vision of a precise medical model projected onto our surroundings such as a holographic human. A transparent view of skin and bone is possible. A holographic human can be moved by the user's hand gestures and interactive manipulation is possible.

\subsection{Navigation function}

A holographic human and 3D-CG surgical instrument can be displayed simultaneously. The movement of 3D-CG surgical instruments can be linked with actual surgical instruments in the operation room. The holographic human is superimposed on the actual patient position. Since the precise positional relationship between the holographic human and surgical instruments can be grasped, 
useful surgical navigation to be possible. The precise position of the actual surgical instruments is represented accurately by HoloLens using the AR marker for Vuforia.

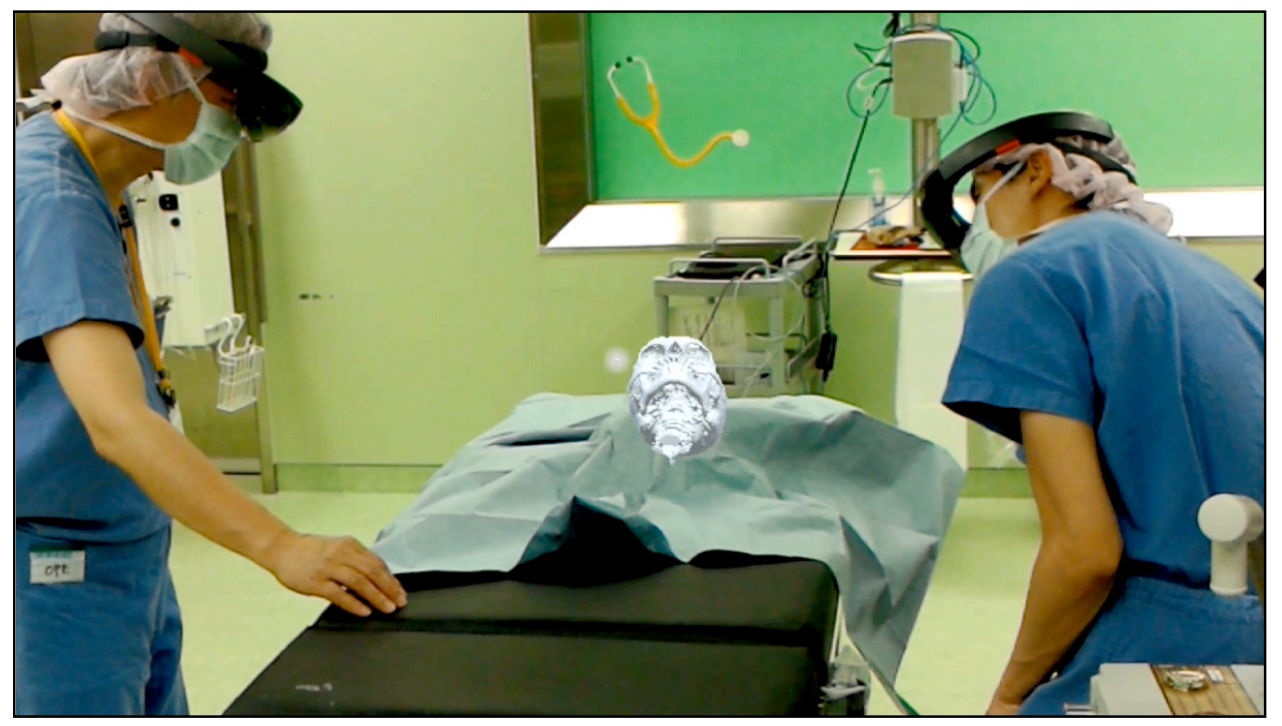

Figure 3: A use case by surgeons

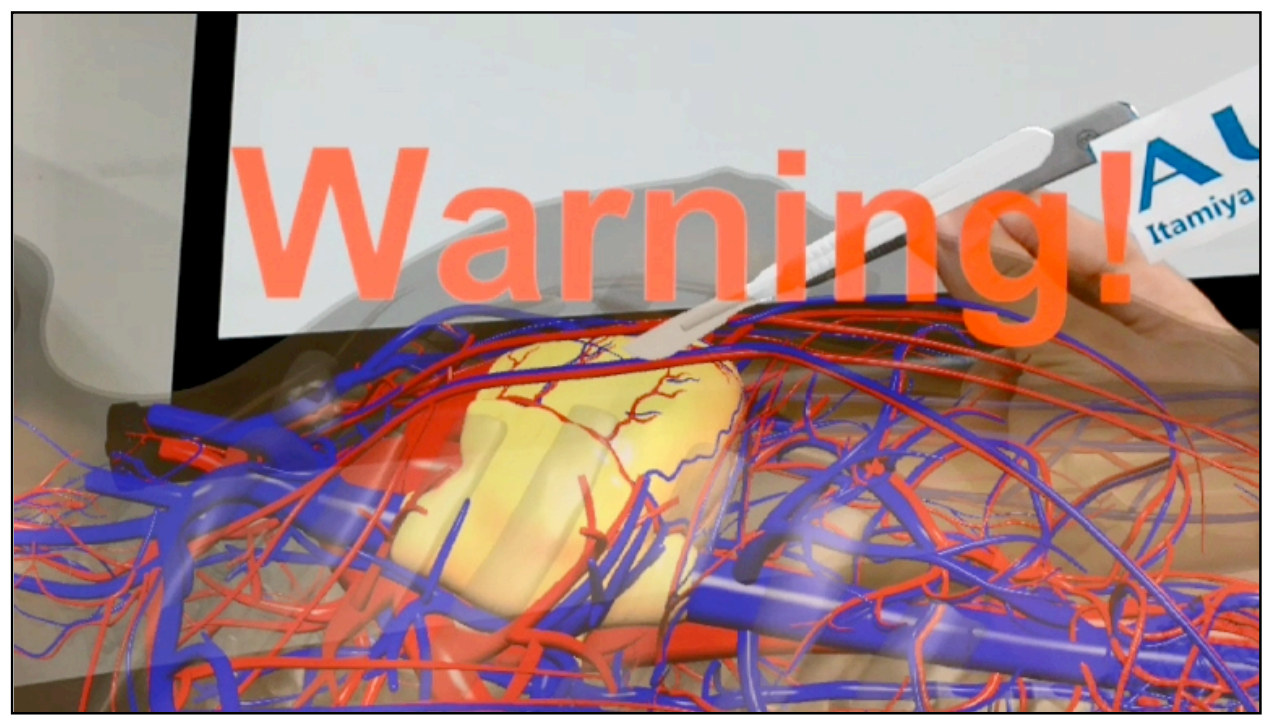

Figure 4: Holographic human and 3D-CG surgical instrument with Warning function to avoid contact with important organs 


\subsection{Multiple persons viewing}

Multiple persons can see one holographic human and 3D-CG surgical instruments at the same time using multiple HoloLenses.

\section{Acknowledgments}

Informed consent was received for publication of patient's picture and 3D-CG medical model in this article (Figure 1 and 3). The 3D model data of Figure 4 used BodyParts3D, (C) Database Center for Life Science, licensed under CC BY-SA 2.1 JP.

\section{References}

[1] BRAINLAB, 2016. Adaptive Hybrid Surgery. https://www.brainlab.com

[2] Mischkowski, R., Zinser, M. J., and Kubler, A., et al. 2006. Application of an augmented reality tool for maxillary positioning in orthognathic surgery - A feasibility study. Journal of Cranio-Maxillofacial Surgery. 34, 478-483.

[3] Badiali, G., Ferrari, V., and Cutolo, F., et al. 2014. Augmented reality as an aid in maxillofacial surgery: Validation of a wearable system allowing maxillary repositioning. Journal of CranioMaxillo-Facial Surgery. 42, 1970-1976.

[4] Suenaga, H., Hoang Tran, H., and Liao, H., et al. 2015. Vision-based markerless registration using stereo vision and an augmented reality surgical navigation system: a pilot study. BMC Medical Imaging. 15:51.

[5] Zhu, M., Chai, G., and Lin, L., et al. 2016. Effectiveness of a Novel Augmented Reality-Based Navigation System in Treatment of Orbital Hypertelorism. Annals of Plastic Surgery. 77(6):662-668.

[6] Itamiya, T., 2016. Augmented Reality using the Binocular Transparent Smart Glasses for Surgical Navigation. Advanced Digital Technology in Head and Neck Reconstruction (ADT) North America \& Japan Regional Leadership Groups Conference in San Diego, 16.

[7] Microsoft, 2016. HoloLens. https:/www.microsoft.com/microsoft-hololens

[8] Materialize, 2016. Mimics. http://www.materialise.com/en/medical $/$ software $/ \mathrm{mimics}$

[9] Unity, 2016. Unity5.5. https://unity3d.com/

[10] PTC, 2016. Vuforia. https://www.vuforia.com/ 